\title{
CF airway smooth muscle transcriptome reveals a role for PYK2
}

\author{
Daniel P. Cook, ${ }^{1,2}$ Ryan J. Adam, ${ }^{3}$ Keyan Zarei, ${ }^{3}$ Benjamin Deonovic, ${ }^{4}$ Mallory R. Stroik, ${ }^{1}$ \\ Nicholas D. Gansemer, ${ }^{1}$ David K. Meyerholz, ${ }^{5}$ Kin Fai Au, ${ }^{1,4}$ and David A. Stoltz ${ }^{1,2,3,6}$
}

Department of Internal Medicine, ${ }^{2}$ Department of Molecular Physiology and Biophysics, and ${ }^{3}$ Department of Biomedical Engineering, Roy J. and Lucille A. Carver College of Medicine, University of lowa, lowa City, lowa, USA. ${ }^{4}$ Department of Biostatistics, College of Public Health, University of lowa, lowa City, lowa, USA. ${ }^{5}$ Department of Pathology, Roy J. and Lucille A. Carver College of Medicine, University of lowa, lowa City, lowa, USA. ${ }^{6}$ Pappajohn Biomedical Institute, University of lowa, lowa City, lowa, USA.

\begin{abstract}
Abnormal airway smooth muscle function can contribute to cystic fibrosis (CF) airway disease. We previously found that airway smooth muscle from newborn CF pigs had increased basal tone, an increased bronchodilator response, and abnormal calcium handling. Since CF pigs lack airway infection and inflammation at birth, these findings suggest intrinsic airway smooth muscle dysfunction in CF. In this study, we tested the hypothesis that CFTR loss in airway smooth muscle would produce a distinct set of changes in the airway smooth muscle transcriptome that we could use to develop novel therapeutic targets. Total RNA sequencing of newborn wild-type and CF airway smooth muscle revealed changes in muscle contraction-related genes, ontologies, and pathways. Using connectivity mapping, we identified several small molecules that elicit transcriptional signatures opposite of CF airway smooth muscle, including NVP-TAE684, an inhibitor of prolinerich tyrosine kinase 2 (PYK2). In CF airway smooth muscle tissue, PYK2 phosphorylation was increased and PYK2 inhibition decreased smooth muscle contraction. In vivo NVP-TAE684 treatment of wild-type mice reduced methacholine-induced airway smooth muscle contraction. These findings suggest that studies in the newborn CF pig may provide an important approach to enhance our understanding of airway smooth muscle biology and for discovery of novel airway smooth muscle therapeutics for CF and other diseases of airway hyperreactivity.
\end{abstract}

Conflict of interest: The authors have declared that no conflict of interest exists.

Submitted: June 5, 2017 Accepted: July 27, 2017 Published: September 7, 2017

Reference information: JCI Insight. 2017;2(17):e95332. https://doi.org/10.1172/jici. insight.95332.

\section{Introduction}

There is increasing awareness that an asthma-like phenotype is present in some people with cystic fibrosis (CF), often termed "CF asthma." Whether dysfunction of the CF transmembrane conductance regulator (CFTR) anion channel contributes directly to the development of CF asthma is not clear. However, several lines of evidence suggest that this might be the case. First, a recent meta-analysis observed an increased risk for asthma among carriers of CFTR mutations compared with noncarriers (1). Second, acute CFTR potentiation, in people with CF and the G551D-CFTR mutation, corrected smooth muscle abnormalities with improvements in airway distensibility and vascular tone (2). Finally, investigations by our group and others have shown that loss or inhibition of CFTR function in animal models can affect smooth muscle tone (2-11). Collectively, these studies indicate that the asthma-like symptoms experienced by individuals with CF could be, in part, due to functional loss of CFTR.

We recently reported that, in porcine airway smooth muscle cells, CFTR localizes to the sarcoplasmic reticulum and that airway smooth muscle from newborn CF pigs has increased contractile tone and disrupted calcium handling (11). Because CF pigs lack airway inflammation at birth (12), these data indicate that loss of CFTR causes a primary defect in airway smooth muscle cell function.

In many cell types involved in airway narrowing, calcium plays an important regulatory role and can act through excitation-transcription coupling to modulate signal transduction pathways and subsequently gene transcription (13). Previous studies have identified transcriptional changes related to airway hyperresponsiveness (14-16). However, cell types other than smooth muscle (e.g., immune and epithelial cells) may have driven both transcriptional changes and airway hyperresponsiveness in these prior studies. Therefore, studies in the CF pig, in which airway smooth muscle hyperresponsiveness is a primary defect, may lead to 


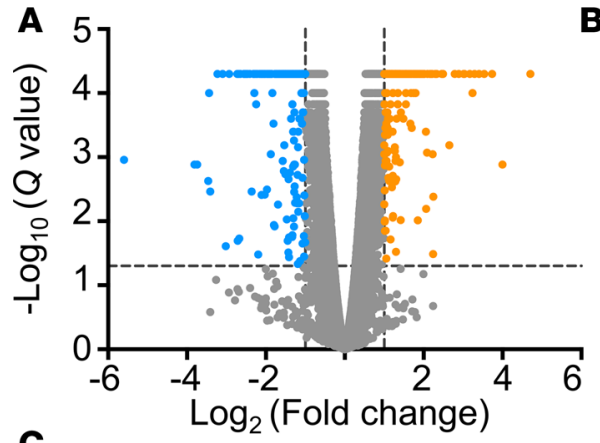

B
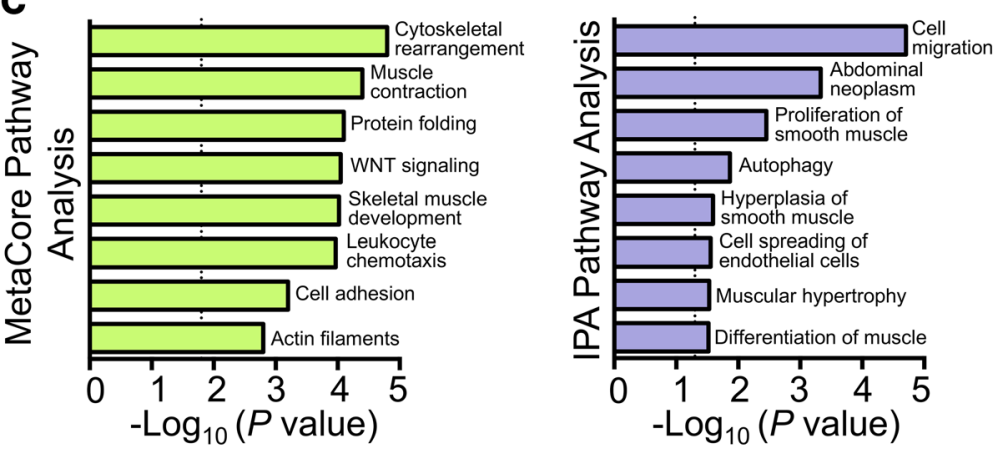

D
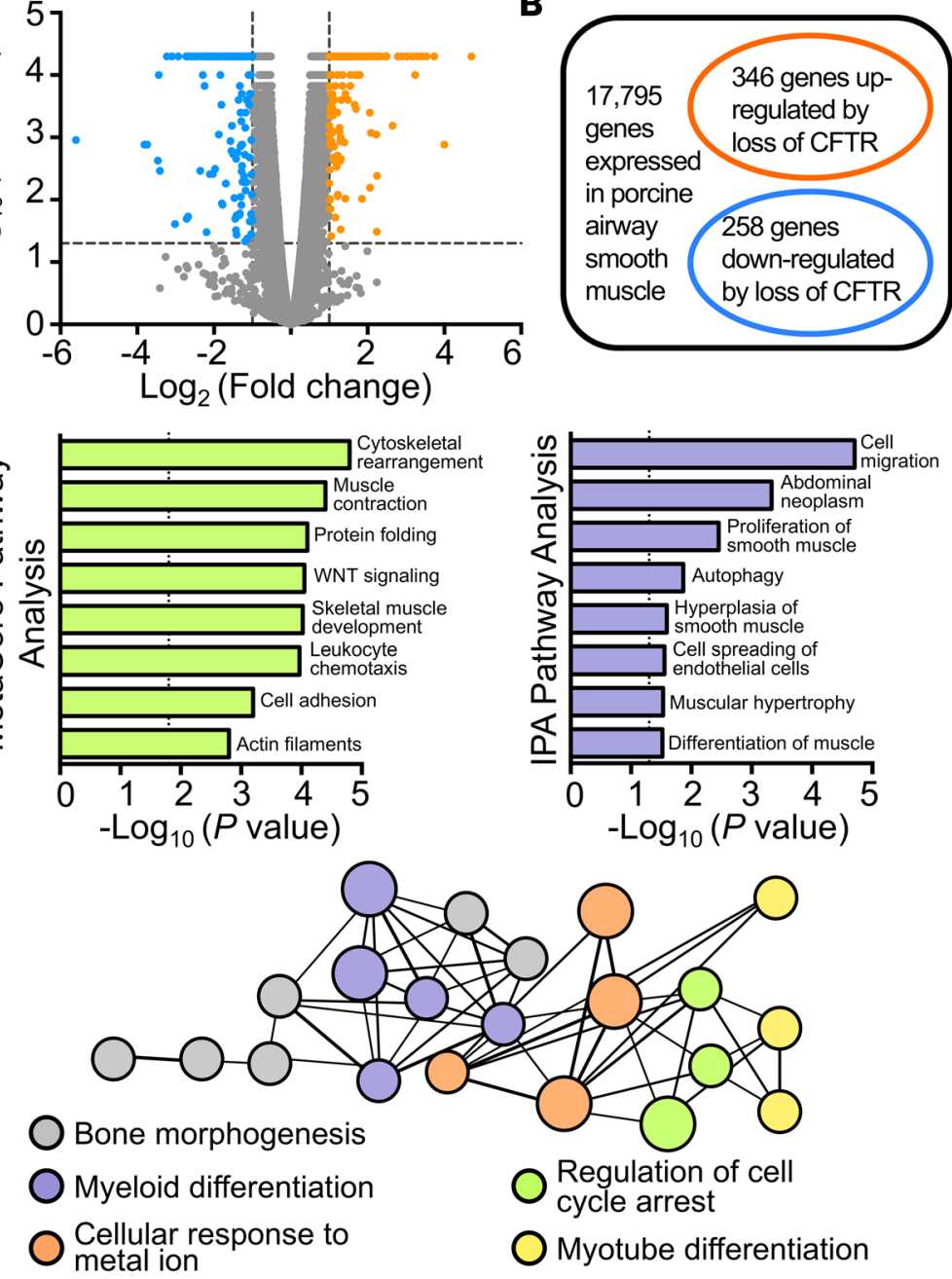

Figure 1. Loss of CFTR leads to alterations in the airway smooth muscle transcriptome. (A) Volcano plot showing differentially expressed genes due to CFTR loss in airway smooth muscle. The negative $\log _{10}$-transformed $\underline{Q}$ values are plotted against the average $\log _{2}$ fold changes in gene expression. Data for genes that were not classified as differentially expressed are plotted in gray. Data for genes that are differentially expressed due to CFTR loss $(\underline{Q}<0.05)$ with an absolute $\log _{2}$ fold change of less than or equal to 1 are denoted by blue symbols and those with an absolute $\log _{2}$ fold change of greater than or equal to 1 are denoted by orange symbols. (B) Summary of gene changes in airway smooth muscle due to CFTR loss. (C) Gene set enrichment analysis for genes that were classified as upregulated or downregulated due to CFTR loss in airway smooth muscle. Data derived from MetaCore enrichment are represented in green, and data derived from Ingenuity Pathway Analysis (IPA) are represented in purple. Both databases were used to generate a list of the top 8 dysregulated pathways represented by the CFTR $^{-1}$ airway smooth muscle signature. Dashed lines represent threshold cutoff for significance for each database. (D) A functionally grouped network of enriched categories was generated for targets altered due to CFTR loss in airway smooth muscle using gene ontology terms as nodes; these terms were linked using ClueGO analysis.

a better understanding of $\mathrm{CF}$ asthma and other diseases of airway hyperreactivity.

In this study, we tested the hypothesis that CFTR loss in newborn pig airway smooth muscle would produce a distinct set of changes in the airway smooth muscle transcriptome. We were able to use the CF airway smooth muscle transcriptional signature and connectivity mapping to generate a list of small molecules predicted to reverse airway smooth muscle dysfunction and to identify a role for proline-rich tyrosine kinase 2 (PYK2) in airway smooth muscle biology and CF airway disease. These findings suggest that the CF pig may represent an important model for understanding the role of CFTR in airway smooth muscle and represent a unique tool for developing therapeutic targets for airway smooth muscle dysfunction.

\section{Results}

Loss of CFTR causes differential gene expression in porcine airway smooth muscle. Previously, we investigated airway smooth muscle from $C F T R^{-/-}$pigs and found an abnormal contractile phenotype and a disruption in calcium handling (11). Because calcium regulates gene transcription, we hypothesized that CFTR loss causes transcriptional changes in airway smooth muscle. To test this hypothesis, we used laser-capture microdissection to isolate airway smooth muscle from $C F T R^{+/+}$and $C F T R^{-/-}$newborn pigs and performed RNA-sequencing (RNA-seq) analysis. Next-generation sequencing and gene analysis identified 604 differentially expressed genes between $\mathrm{CFTR}^{+/+}$and $\mathrm{CFTR}^{-/-}$airway smooth muscle (Figure $1, \mathrm{~A}$ and $\mathrm{B}$, and Supplemental Table 1; supplemental material available online with this article; https://doi.org/10.1172/ jci.insight.95332DS1). Some of these differentially expressed genes have important roles in airway smooth muscle contractile function, including ROCK1 (Rho associated coiled-coil containing protein kinase 1), MYLK (myosin light chain kinase), CALM6 (calmodulin-6), and MYL6 (myosin light chain 6) (17). Quantitative RT-PCR was used to validate several of the mRNA transcript changes in airway smooth musclerelated genes (Supplemental Table 2).

Gene set enrichment analysis, using MetaCore and Ingenuity Pathway Analysis (IPA), identified functional networks overrepresented within the 604 genes (Figure 1C). The top two enriched networks included gene ontology $(\mathrm{GO})$ terms related to cytoskeletal rearrangement/muscle contraction and cell migration/ 

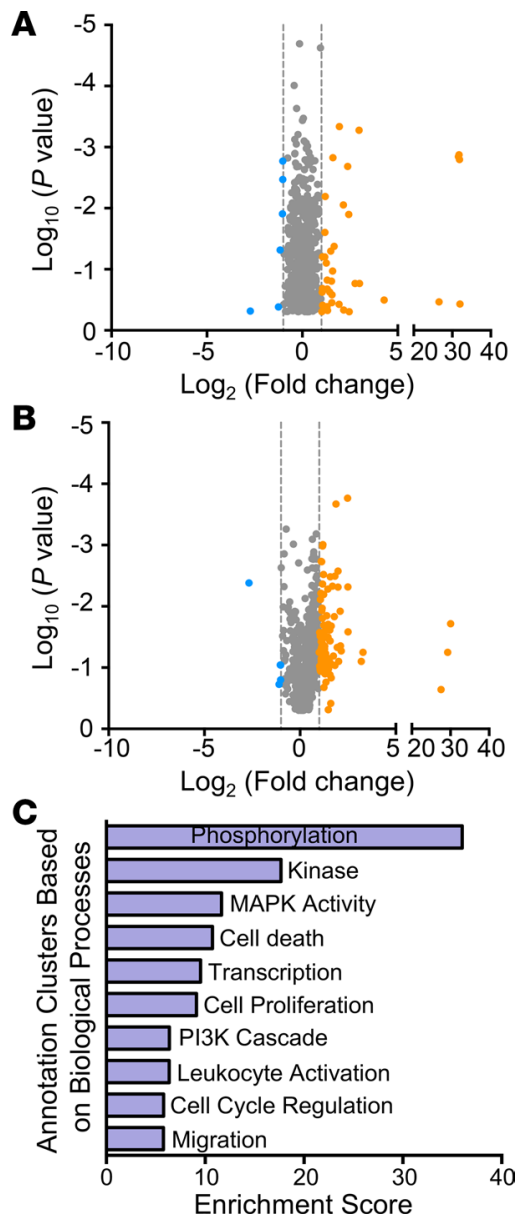

Enrichment Score
Figure 2. Proteomic changes parallel transcriptional alterations in response to CFTR loss. (A) Volcano plot of total protein expression, as identified by a targeted antibody array in CFTR ${ }^{-1-}$ airway smooth muscle cells compared with WT cells. Each dot represents a protein-specific site. The dashed lines represent a 2-fold change from WT levels. Proteins that expressed at least 2-fold less than (blue) or greater than (orange) WT are shown. (B) Volcano plot of protein phosphorylation status, as identified by a targeted antibody array in $\mathrm{CFTR}^{-/-}$airway smooth muscle cells compared with WT cells. Each dot represents a phosphorylation site. The dashed lines represent a 2-fold change from WT levels. Proteins with phosphorylation changes at least 2-fold less than (blue) or greater than (orange) WT are shown. (C) Enrichment analysis of the upregulated and downregulated proteins in addition to proteins with altered phosphorylation status.

abdominal neoplasm. Other enriched networks between the two databases that could be related to smooth muscle biology included skeletal muscle development, proliferation of smooth muscle, hyperplasia of smooth muscle, muscular hypertrophy, differentiation of muscle, and actin filaments. These enrichments indicate that CFTR loss causes mRNA expression changes not only related to the contractile machinery, but also to cell regulatory pathways.

IPA can identify potential upstream regulatory nodes by interrogating the downstream consequences of a specific node (Ingenuity Systems; https://analysis.ingenuity.com/pa). Using this analysis, we found 9 upstream regulators that were dysregulated due to CFTR loss (Supplemental Table 3). Interestingly, many of the proposed upstream regulators, such as phosphatidylinositol-4,5-bisphosphate 3-kinase (PI3K) and phosphodiesterase signaling, have been implicated in airway smooth muscle function and disease $(18,19)$. Others, such as WNT and CREB, have been studied in CF (20-22).

To further probe the differentially expressed gene networks in $\mathrm{CFTR}^{-/-}$airway smooth muscle, we used the ClueGo plug-in of Cytoscope 3.1.0 (23). This analysis tool aggregates individual gene enrichment pathways with a functionally arranged GO term network. The result is a functional network that shows the relations between GO terms based upon their similarity and connectivity. Using our RNA-seq data set, we found that the major network related to biological process was associated with terms involved with cell differentiation and responses to metal ions such as calcium (Figure 1D). Collectively, these data suggest that five major groups of biological processes (bone morphogenesis, myeloid differentiation, cellular response to metal ion, regulation of cell cycle arrest, and myotube differentiation) are altered in $C F T R^{-/-}$airway smooth muscle. These results indicate that CFTR loss in airway smooth muscle can lead to transcriptional changes outside of the canonical muscle machinery.

Protein analysis of CF airway smooth muscle shows pathway dysregulation similar to the CFTR ${ }^{-1-}$ transcriptome. To investigate whether proteomic changes were reflective of transcriptional network alterations, we performed a proteomic screen using an antibody array to identify proteins differentially regulated in $C F T R^{-/-}$ airway smooth muscle cells (24). The array contains approximately 1,300 antibodies against individual proteins and their phosphorylation sites, with some proteins represented by multiple antibodies, encompassing over 30 different regulatory pathways. Comparing whole-cell lysates from WT and $C F T R^{-/-}$airway smooth muscle, we found 43 proteins with greater than 2-fold change between $C F T R^{+/+}$and $C F T R^{-/-}$airway smooth muscle (6 sites comprising 6 different proteins down and 39 sites comprising 37 proteins up, Figure 2A). When we compared the differential protein expression to the differential transcript expression, we observed a significant positive correlation (Supplemental Figure 1). These results indicate that loss of CFTR produces transcript changes that subsequently alter protein expression.

CFTR loss altered 93 phosphorylation sites greater than 2-fold (5 sites had decreased phosphorylation and 88 sites had increased phosphorylation, Figure 2B). Enrichment analysis of proteins dysregulated in total abundance and phosphorylation indicated that "phosphorylation" and "kinase" were the most enriched terms. This result was expected, given that the array used was specifically designed to detect phosphorylated proteins. However, consistent with our findings at the transcriptional level, other pathways reflecting MAPK activity, cell death, transcription, cell proliferation, and the PI3K cascade were also dysregulated (Figure $2 \mathrm{C}$ ).

Connectivity mapping identified compounds that elicit an $m R N A$ expression signature opposite of that observed in CFTR-deficient airway smooth muscle. Since we found broad-scale changes in the transcriptome of $C F T R^{-/-}$ airway smooth muscle, we predicted that we could use these transcriptional alterations to identify novel therapeutics for airway smooth muscle dysfunction (25) (Figure 3A). To test this hypothesis, we looked 
A

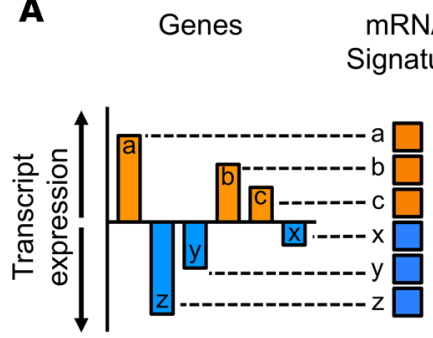
mRNA Signature

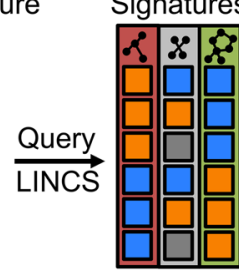

Select Reversing Compounds

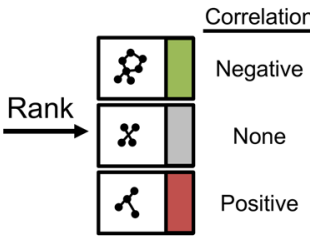

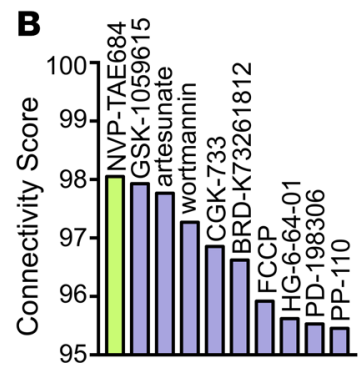

C

Likelihood of top ranked compounds association with disease state

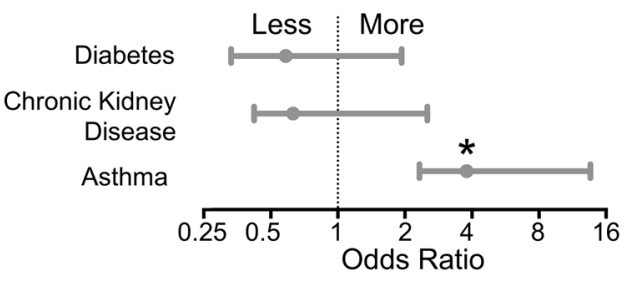

Figure 3. Connectivity mapping can be used to generate therapeutic targets based upon the CF airway smooth muscle transcriptional signature. (A) Overview of approach. Transcriptional profiles from two distinct groups (denoted by blue and orange in this schematic; for our study WT and CFTR ${ }^{-1}$ porcine airway smooth muscle) are queried against the LINCS database, which contains compound signatures. Compounds are ranked based upon the direction and strength of enrichment with the transcriptional profile of $\mathrm{CFTR}^{-/-}$airway smooth muscle to generate a list of positively and negatively correlated compounds. (B) From this nonbiased approach, a list of potential candidate small molecules that elicit a transcriptional signature negatively correlated to the CF airway smooth muscle transcriptome was generated. (C) The odds ratio of an association for LINCS-generated compounds from the CFTR ${ }^{-/-}$airway smooth muscle gene signature with the PubMed database terms "asthma," "chronic kidney disease," and "diabetes" was determined (see Methods for further details). Data are plotted with mean and 95\% confidence intervals. "Diabetes" and "chronic kidney disease" were used as non-airway smooth muscle-mediated disease controls.

for small molecules whose transcriptional signature negatively correlated to the $C F T R^{-/-}$airway smooth muscle signature. We used the Library of Integrated Network-Based Cellular Signatures (LINCS) to compare the $C F T R^{-1-}$ airway smooth muscle mRNA expression signature to the transcriptional signatures elicited by treatment of approximately 150 cell lines with hundreds of small-molecule compounds.

With this approach, we generated a list of potential candidate small molecules that elicited a transcriptional signature negatively correlated to $\mathrm{CFTR}^{-/-}$airway smooth muscle (Figure $3 \mathrm{~B}$ ). One of the small-molecule compounds with a high-connectivity score was GSK1059615, a pan PI3K inhibitor. Inhibition of PI3K has been shown to reduce airway remodeling $(26,27)$, reduce ovalbumin-induced airway hyperresponsiveness in mice (28), and reverse carbachol-induced constriction of human airways (18). The presence of this airway-modifying drug on our list of potential compounds supported our hypothesis that connectivity mapping could be used to identify therapeutics for airway smooth muscle dysfunction.

To assess the specificity of our findings, as applied to asthma, we performed a literature review using the PubMed database and screened for compound associations with the disease MeSH term "asthma." In total, when analyzing the 60 compounds with the highest connectivity scores, we found an enrichment of compounds that have associations with the MeSH term asthma, as compared with a list of 60 compounds showing little or no connectivity (Figure 3C). A similar analysis was performed using two MeSH terms unrelated to airway smooth muscle, "diabetes mellitus" and "chronic kidney disease." The 60 top compounds did not show significant associations with either of these terms

Based upon these discovery validations, we decided to pursue the small molecule with the highest connectivity score, NVP-TAE684, a potent anaplastic lymphoma kinase (ALK) and PYK2 inhibitor (29, 30). NVP-TAE684's effects on smooth muscle are unknown. However, the finding that the transcriptional profile of NVP-TAE684 negatively correlated with the $C F T R^{-/-}$airway smooth muscle signature suggested that the small molecule might inhibit airway smooth muscle contraction.

PYK2 is expressed in airway smooth muscle. Since NVP-TAE684 has been shown to inhibit both PYK2 and ALK, we next determined whether airway smooth muscle expresses $P Y K 2$ and/or $A L K$ mRNA in situ. Using the RNA-seq data, we found that $P Y K 2$ transcripts were present in both WT and $C F T R^{-/-}$airway smooth muscle, while $A L K$ transcript levels were below our quantitative threshold (Figure $4 \mathrm{~A}$ ). To confirm these findings, quantitative RT-PCR was performed and revealed that $P Y K 2$ transcripts were present in airway smooth muscle and similar levels were present between WT and $C F T R^{-/-}$airway smooth muscle (Figure 4, B and C). Immunocytochemistry of cultured airway smooth muscle cells showed positive immunostaining for PYK2 (Figure 4D). Collectively, these data show that PYK2 is expressed at both the transcript and protein levels in airway smooth muscle.

PYK2 blockade inhibits cholinergic-induced airway smooth muscle contraction. Knowing that CFTR $^{-/-}$airway smooth muscle displays increased contractile tone and transcriptional profile changes, we hypothesized that PYK2 inhibition would prevent airway smooth muscle contraction. To confirm that NVP-TAE684 inhibits PYK2, we first added NVP-TAE684 to recombinant PYK2 and observed a nearly $75 \%$ reduction in PYK2 activity (Figure 5A). We next tested the effect of PYK2 inhibition on airway smooth muscle contraction in lung slices from WT newborn pigs. Compared with lung slices pretreated with DMSO, methacholine-induced 

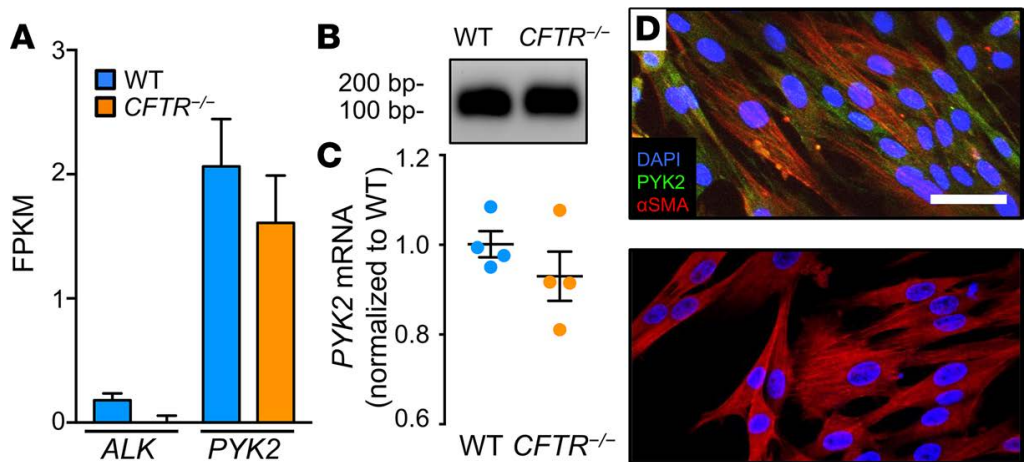

Figure 4. PYK2 but not $A L K$ is present in airway smooth muscle. (A) Relative expression of PYK2 and $A L K$ in WT and CFTR ${ }^{-/}$airway smooth muscle tissue measured by RNA-seq in fragments per kilobase of transcript per million mapped reads (FPKM). For each group, data are the computationally derived mean and SD from $n=4$ donors per group, using Cufflinks analysis. Relative expression of PYK2 in WT and CFTR ${ }^{-1}$ airway smooth muscle tissue, as shown in (B) DNA gel and (C) qPCR measurements. $n=4$ donors per group. The horizontal lines represent the mean and error bars denote SEM. (D) PYK2 protein expression in WT airway smooth muscle cells. PYK2 immunostaining was performed in WT airway smooth muscle cells (top). Rabbit polyclonal lgG isotype antibody control is also shown (bottom). Blue staining (DAPI), red staining ( $\alpha$-smooth muscle actin), and green staining (PYK2). Scale bar: $40 \mu \mathrm{m}$.

airway narrowing was significantly $(P<0.05)$ reduced in NVP-TAE684-treated lung slices (Figure 5B). These results indicate that NVP-TAE684 can decrease cholinergic-induced airway contractility in porcine lung slices.

We hypothesized that NVP-TAE684's effects were, in part, due to PYK2 inhibition in airway smooth muscle cells. Since precision-cut lung slices possess numerous cell types, including chondrocytes, airway epithelial cells, and smooth muscle cells (31), we tested NVP-TAE684's effects directly on tracheal smooth muscle in the absence of airway epithelial cells. Isolated tracheal smooth muscle samples from WT pigs pretreated with NVP-TAE684 generated less force than DMSO-pretreated control samples (Figure 5C). The $\mathrm{EC}_{50}$ for acetylcholine was similar between control and NVP-TAE684 groups, suggesting that the effect of PYK2 inhibition is unlikely due to competitive effects on cholinergic binding at the muscarinic receptor.

PYK2 inhibition reduces myosin light chain phosphorylation. To investigate if PYK2 inhibition causes alterations in the contractile machinery of airway smooth muscle cells, we quantified regulatory myosin light chain (MLC) phosphorylation. Increased MLC phosphorylation is required for actin-myosin interactions, leading to airway smooth muscle contraction. NVP-TAE684 treatment significantly diminished acetylcholine-induced MLC phosphorylation compared with that in DMSO control samples (Figure 6, A and B). These results suggest that PYK2 is an upstream regulator of MLC phosphorylation and PYK2 inhibition decreases MLC phosphorylation.
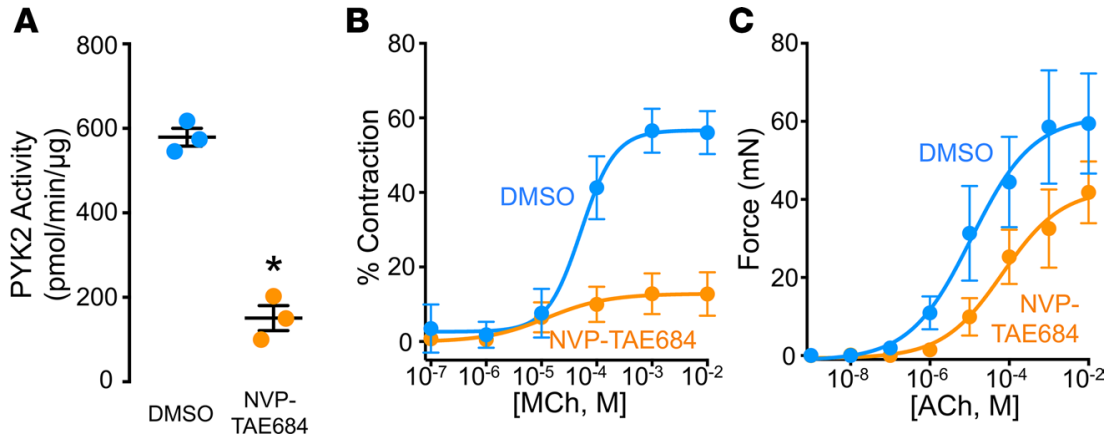

Figure 5. PYK2 inhibition decreases airway smooth muscle contraction. (A) Recombinant human PYK2 was treated with either DMSO or $1 \mu \mathrm{M}$ NVPTAE684. PYK2 activity was measured by using an ADP-based phosphatase-coupled kinase assay. ADP from PYK2 activity releases an inorganic phosphate via a coupling enzyme and is detected using malachite green reagent. The amount of inorganic phosphate released is proportional to the amount of ADP generated during the kinase reaction. $n=3$ reactions per group; unpaired Student's $t$ test was used for analysis. Individual data points are shown, horizontal lines represent the mean, and error bars denote SEM. ${ }^{*} P<0.05$. (B) Percentage airway lumen area narrowing (\% contraction) in response to methacholine (MCh) in WT precision-cut lung slices pretreated with control vehicle (DMSO, blue circles) or NVP-TAE684 (orange circles) normalized to maximal contractile response ( $n=6$ animals per group). (C) WT tracheal smooth muscle strip isometric force generation following acetylcholine (ACh) treatment in muscle strips pretreated with control vehicle (DMSO, blue circles) or NVP-TAE684 (orange circles) ( $n=5$ animals per group). Data are shown as mean values with SEM and are fitted with a 4-parameter logistic regression algorithm (solid line) for $\mathbf{B}$ and $\mathbf{C}$. $P<0.05$ for curve comparisons between groups in $\mathbf{B}$ and $\mathbf{C}$. 
A

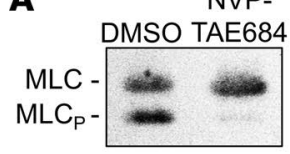

B

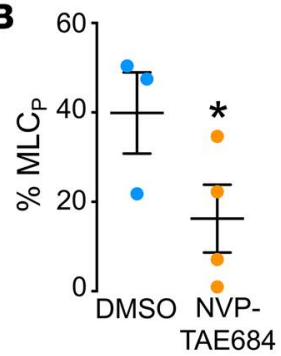

D
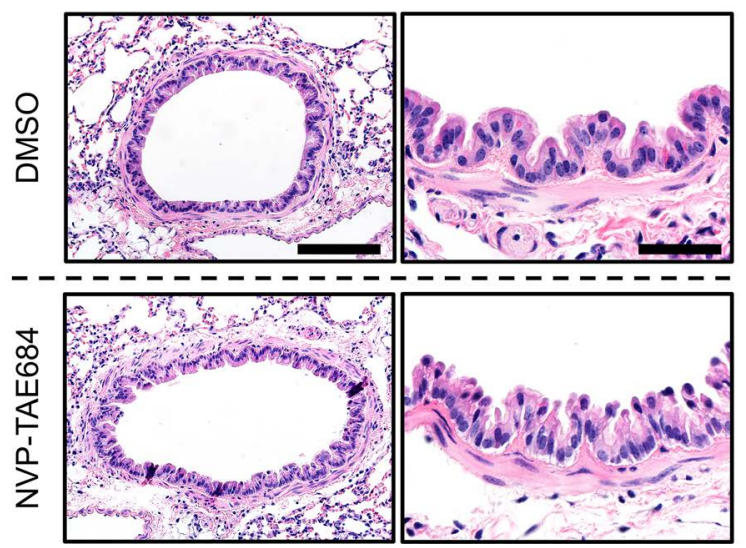

Figure 6. PYK2 inhibition decreases myosin light chain phosphorylation and cholinergic-induced airway narrowing. (A) Immunoblot for total and phosphorylated regulatory myosin light chain (MLC and LLC $_{\mathrm{p}}$, respectively) in DMSO and NVP-

TAE684 WT airway smooth muscle cells. (B) Quantification of percentage myosin light chain phosphorylation in WT airway smooth muscle cells from DMSO- or NVP-TAE684-treated samples ( $n=3$ donors per group). Individual data points are shown, horizontal lines represent the mean, and error bars denote SEM. Unpaired Student's $t$ test was used for analysis. ${ }^{*} P<0.05$. (C) Newtonian lung resistance following methacholine (MCh) treatment in WT mice pretreated with control vehicle (DMSO, blue circles) or NVP-TAE684 (orange circles) ( $n=10$ animals per group). Data are shown as mean values with SEM and are fitted with a 4-parameter logistic regression algorithm (solid line). $P<0.05$ for curve comparisons between groups. (D) Histological $\mathrm{H} \& \mathrm{E}$ section of an airway with surrounding lung parenchyma from DMSO and NVP-TAE684-treated mice following methacholine challenge. Scale bar: $100 \mu \mathrm{m}$ (left); $35 \mu \mathrm{m}$ (right).

PYK2 inhibition reduces in vivo cholinergic-induced airway hyperresponsiveness in mice. We next evaluated the in vivo effects of PYK2 inhibition on pulmonary airway reactivity by measuring airway resistance. Wild-type mice received daily intraperitoneal injections of NVP-TAE684 for 3 days prior to conducting in vivo lung resistance measurements. NVP-TAE684 treatment significantly reduced methacholine-induced increases in airway resistance as compared with control mice treated with DMSO (Figure 6C). Morphological analysis of histological sections taken from the lung parenchyma and conducting airways demonstrated that there were no significant histological changes caused by NVP-TAE684 treatment (Figure 6D). These findings indicate that (a) PYK2 inhibition can prevent cholinergic-induced airway reactivity in vivo and (b) the LINCS approach to novel compound discovery using a porcine model can identify targets across species.

PYK2 inhibition reverses the CF-induced airway hyperresponsive phenotype. It has previously been shown that PYK2 phosphorylation correlates with PYK2 kinase activity (32). Full activation of PYK2 requires phosphorylation at the autophosphorylation site Tyr-402 (33). We therefore asked whether there was increased PYK2 phosphorylation in $C F T R^{-/-}$airway smooth muscle cells. Protein lysates from WT, $C F T R^{-/-}$, and NVP-TAE684-pretreated $C F T R^{-/-}$airway smooth muscle cells were collected, and we used an ELISA-based antibody array to quantify total abundance and Tyr-402 phosphorylation status of PYK2. PYK2 phosphorylation was increased in $C F T R^{-/-}$airway smooth muscle cells, and NVP-TAE684 pretreatment reduced PYK2 phosphorylation to WT levels (Figure 7A). These data were confirmed using Western blot analysis of WT and $C F T R^{-/-}$airway smooth muscle cell lysates (Supplemental Figure 2).

Simultaneously, the downstream targets of PYK2, the signaling proteins PI3K and MAPK, were found to have increased phosphorylation in $C F T R^{-/-}$compared with WT airway smooth muscle cells (Figure 7, B and C). PYK2 inhibition reduced PI3K and MAPK phosphorylation to levels, such that they were comparable to those of WT airway smooth muscle cells. These results suggest that NVP-TAE684 works via inhibition of PYK2 autophosphorylation and subsequent inhibition of the PYK2 signaling cascade, including activation of PI3K and MAPK signaling pathways.

Finally, to investigate the effects of PYK2 inhibition on $C F T R^{-/-}$airway smooth muscle contraction, we measured tracheal muscle force generation. Previously, we described an airway hyperresponsive phenotype in newborn $C F T R^{-/-}$piglets in the absence of inflammation and infection. To determine if PYK2 inhibition could reverse this phenotype, we used WT and $C F T R^{-/-}$airway smooth muscle. In order to achieve comparable resting tone, WT and $C F T R^{-/-}$airway smooth muscles were pretreated with isoproterenol. Acetylcholine caused greater force production in $C F T R^{-/-}$tracheal smooth muscle compared with WT muscle (Figure 7D). This is consistent with previously reported findings of increased contractile tone in $C F T R^{-/-}$airway smooth muscle (11). Pretreatment with NVPTAE684 decreased force generation in $\mathrm{CFTR}^{-/-}$airway smooth muscle strips (Figure 7D). There were no significant differences in the $\mathrm{EC}_{50}$ of acetylcholine among the three groups. These results suggest that PYK2 inhibition can partially rescue the contractile phenotype induced by CFTR loss, in part, through inhibition of airway smooth muscle PYK2. 
A
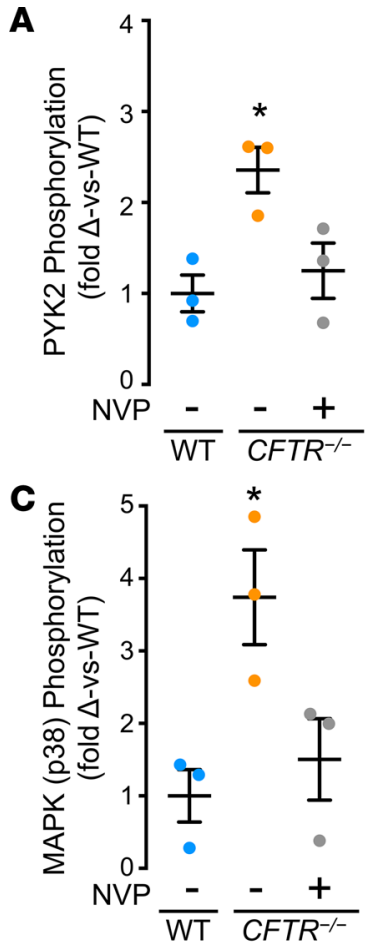

B
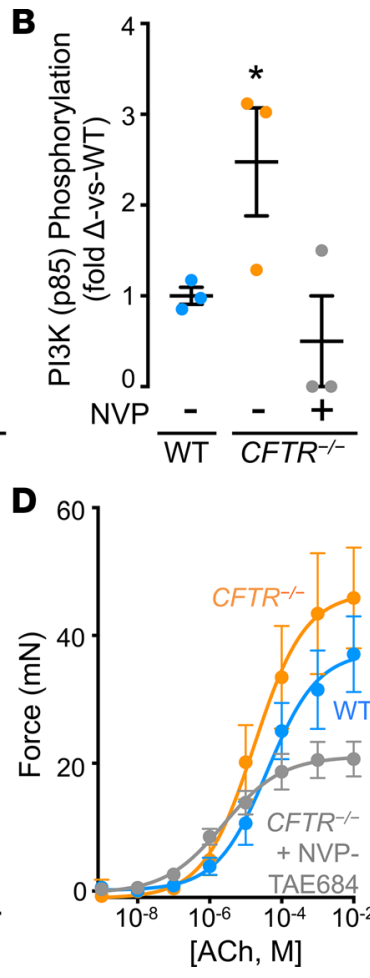

Figure 7. CFTR ${ }^{-/}$airway smooth muscle has altered PYK2 and downstream target phosphorylation. Airway smooth muscle cell lysates from WT, CFTR ${ }^{-1-}$, and NVPTAE684-pretreated CFTR $^{-/}$cells were compared on an ELISA containing immobilized antibodies to unphosphorylated and phosphorylated (A) PYK2; (B) PI3K regulatory subunit p85; and (C) MAPK regulatory subunit p38. Percentage phosphorylation for each protein was calculated from the phosphorylated and the total protein fluorescent intensity. Data are shown as the fold change $(\Delta)$ versus WT mean with SEM (a total of 4 donors/genotype were pooled onto one antibody microarray slide and a minimum of 3 slides/genotype were run, thus $n=3$ slides, representing 12 animal donors/genotype). One-way ANOVA followed by Dunnett's test was used for statistical analysis in A-C. ${ }^{*} P<0.05$. (D) Isoproterenol prerelaxed WT and $C F T R^{-/-}$airway smooth muscle strip isometric force generation following acetylcholine (ACh) treatment in tracheal muscle strips. CFTR ${ }^{-1}$ airway smooth muscle strips were pretreated with control vehicle (DMSO, orange symbols) or NVP-TAE684 (gray circles), and control WT strips were pretreated with DMSO (blue symbols) ( $n=5$ animals per group). Data are shown as mean values with SEM and are fitted with a 4-parameter logistic regression algorithm (solid line). $P$ $<0.05$ for curve comparisons between all groups in $\mathbf{D}$. $n=7-11$ donors per group.

\section{Discussion}

Our findings suggest that the newborn CF pig, which lacks airway inflammation, represents a model to study intrinsic airway smooth muscle dysfunction. Our previous studies have shown that CFTR loss increases airway smooth muscle tone and alters airway reactivity (11). Our current data expand upon those studies by providing a whole-transcriptome assessment of airway smooth muscle from $\mathrm{CF}$ and WT newborn pigs. In the current study, (a) we identified a subset of genes that are dysregulated in airway smooth muscle in response to CFTR loss. and global cell signaling. (b) We used the CF transcriptome and connectivity mapping to investigate airway smooth muscle biology and generate possible therapeutic targets, and (c) we found that PYK2 is important in airway smooth muscle function and represents a druggable target in diseases of airway hyperreactivity.

By using laser-capture microdissection and RNA-seq analysis, we were able to examine the effects of CFTR loss in airway smooth muscle on mRNA expression signatures. As predicted, we observed transcriptional changes reflective of smooth muscle contraction biology, including transcriptional expression changes in ROCK1, MYLK, CALM6, and MYL6. An unexpected finding was that CFTR loss induced a large number of transcriptional changes outside of the canonical contractile machinery pathways. In total, there were 604 genes altered in $C F T R^{-/-}$airway smooth muscle. These changes represented many different biological processes and functional pathways but had common associations with smooth muscle activity, cellular signaling, and cell growth.

Connectivity mapping and subsequent phosphorylation studies demonstrated that PYK2 had increased phosphorylation in $\mathrm{CFTR}^{-/-}$airway smooth muscle. PYK2 inhibition reduced airway smooth muscle contraction. Interestingly, in ovalbumin-sensitized mice, PYK2 inhibition decreased inflammation and reduced airway hyperresponsiveness (34). However, that study did not explore the direct effects of PYK2 inhibition on smooth muscle, but instead focused on the inflammatory response. Thus, those observed effects could have been related to decreased airway inflammation, independent of a direct effect on airway smooth muscle function. These data, together, with our findings suggest that, in diseases of airway narrowing and inflammation, PYK2 may have a direct effect on smooth muscle contraction while also decreasing airway inflammation.

Several mechanisms could account for PYK2's effect on airway smooth muscle contractility. Previous studies have shown that calcium can activate PYK2 by causing autophosphorylation at Tyr-402, a site that is required for kinase activity (33). Active PYK2 in vascular smooth muscle has been shown to mediate $\mathrm{Ca}^{2+}$ /calmodulin-dependent PYK2 homodimer formation and transphosphorylation (35) and inhibit MYPT1 through a RhoA/ROCK pathway (36). Moreover, PYK2 inhibition has been shown to prevent stretch-induced activation of Akt and ERK1/2 (37). Given the observed changes in PI3K and MAPK signaling pathways following PYK2 inhibition in airway smooth muscle cells, it is likely that PYK2 is acting, through similar mechanisms, to reduce contractility, as in vascular smooth muscle.

Our results support a proposed model in which CFTR loss in airway smooth muscle dysregulates intracellular calcium, which, in turn, activates the contractile apparatus, including MLC and calcium sensitization 


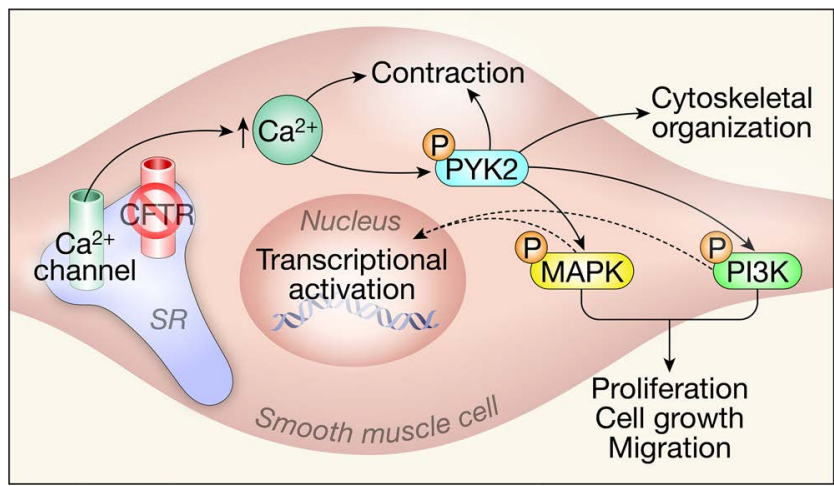

Figure 8. Model of airway smooth muscle alterations in CF. Loss of CFTR leads to alterations in intracellular calcium handling. The increase in $\mathrm{Ca}^{2+}$ can lead to direct changes to the contractile apparatus or lead to PYK2 activation and autophosphorylation. Phosphorylated PYK2 can promote changes in the cytoskeleton and activate PI3K and MAPK signaling pathways. These pathways can lead to changes in proliferation, cell growth, and migration. In addition, activation of transcription factors could lead to gene expression changes. proteins, such as PYK2, and, ultimately, contributes to excitation-transcription coupling (Figure 8). We previously found that loss of CFTR delayed $\mathrm{Ca}^{2+}$ reuptake following a cholinergic stimulus. Others have proposed that chloride channels promote charge neutralization across the sarcoplasmic reticulum membrane during uptake of calcium ions (38). Studies investigating chloride in sarcoplasmic reticulum function (38-42) support a role for CFTR in calcium handling. In this proposed model (Figure 8), the altered calcium ion flux of the CF airway smooth muscle cell activates second messenger pathways, such as PKC, calmodulin, or calcium/calmodulin-dependent kinase II (CAMKII), to cause direct effects in the contractile machinery. In parallel, altered calcium dynamics can lead to global effects on cell proliferation, migration, and transcription through alterations in $\mathrm{Ca}^{2+}$-sensitive proteins (such as PYK2) and $\mathrm{Ca}^{2+}$-induced signaling pathways (such as PI3K, MAPK, WNT). Additionally, PYK2 has been found to play a role in arterial smooth muscle contractile differentiation (43). Future studies will be required to better understand the relative contribution of PYK2 in the hypercontractile phenotype observed due to CFTR loss.

Our studies have both advantages and limitations. Advantages include that (a) the $C F T R^{-/-}$pig recapitulates many aspects of the disease phenotypes seen in human CF $(12,44-47)$. (b) By using newborn $C F T R^{-/-}$pigs, we were able to investigate an intrinsic defect of smooth muscle dysfunction absent inflammation. (c) The combination of laser-capture microdissection and RNA-seq profiling allowed us to develop an unbiased approach to selectively investigate the airway smooth muscle transcriptome using physiologically relevant tissue. (d) By using a transcriptomics approach and the LINCS database, we were able to identify potentially novel targets and compounds that may have therapeutic benefit. Limitations include that (a) we cannot exclude the possibility that developmental changes might contribute to the airway smooth muscle defects in CF pigs. However, previous data from acute CFTR inhibition experiments $(11,48)$, in addition to our current PYK2 inhibition studies, would suggest that the phenotype is, at least in part, due to an intrinsic defect from CFTR loss. (b) While we selectively isolated airway smooth muscle from tracheal sections to develop the transcriptomes, we cannot rule out contributing effects of CFTR in other cell types, such as epithelia or neurons.

In summary, with an integrative approach, combining genetically modified pigs, next-generation transcriptional sequencing, molecular studies, and physiological assays, we have demonstrated that increased phosphorylation of a airway smooth muscle protein, PYK2, contributes to abnormal airway smooth muscle function in CF. This understanding will facilitate further insights into the pathophysiology of airway hyperresponsiveness and therapeutic options for airway smooth muscle dysfunction.

\section{Methods}

Animals. We previously reported the production of $C F T R^{+/-}$and $C F T R^{-/-}$pigs $(49,50)$. Animals were purchased from Exemplar Genetics, and we used WT $\left(\mathrm{CFTR}^{+/+}\right)$and $\mathrm{CF}\left(\mathrm{CFTR}^{-/-}\right)$pigs.

Tissue collection and preservation for laser-capture microdissection. Immediately after euthanasia, tracheas were placed into a plastic cryomold cassette and embedded in optimal cutting temperature compound. A total of 4 tissue samples from each genotype were collected. The samples were placed on dry ice until frozen and stored at $-80^{\circ} \mathrm{C}$ until microdissection.

Laser-capture microdissection. Serial $8-\mu \mathrm{m}$ frozen sections were cut at $-20^{\circ} \mathrm{C}$ using RNAse-free blades and mounted on Arcturus PEN membrane frame slides. These slides were then fixed in serial nuclease-free ethanol washes ranging from $95 \%$ to $50 \%$ ethanol; rehydrated in nuclease-free water for 60 seconds; and dehydrated by sequential immersion into $50 \%, 75 \%, 95 \%$, and $100 \%$ ethanol for 30 seconds each. The ethanol dehydrated slide was washed in xylene for 5 minutes, and then excess xylene was blotted from the slide. Laser-capture microdissection was performed using a Leica LMD7000 Laser Capture Microdissection System (Leica Microsystems). In the newborn pig trachea, the airway smooth muscle layer is easily identified as a uniform layer of cells that runs transverse below the epithelial layer and immediately above the cartilage rings. Using laser-capture microdissection, we were able to capture this layer of cells in its entirety. Tissue was captured from the slides using RNAse-free tubes. Approximately 1,000 cells were captured from the 
airway smooth muscle layer in each slide over approximately 10 minutes, incubated at $42^{\circ} \mathrm{C}$ for 30 minutes in $50 \mu \mathrm{l}$ extraction buffer, and stored at $-80^{\circ} \mathrm{C}$. The microdissected cells from the same donor slides were pooled and used for the RNA extraction.

RNA isolation. Total RNA was isolated from microdissected airway smooth muscle tissue using a PicoPure Kit (Life Technologies) according to the manufacturer's instructions. Genomic DNA was digested using DNase I (Qiagen). RNA samples were then quantified using fluorimetry (Qubit 2.0 fluorometer; Life Technologies), and RNA quality was assessed using an Agilent BioAnalyzer 2100 (Agilent Technologies). Only samples with RNA integrity numbers $>7$ were used.

To validate our RNA-seq findings, qPCR was performed using Takara Bio SYBR Green Master Mix with ROX (Clontech). Primer sequences were as follows: CFTR forward: 5'-CTGGAGCCTTCAGAGGGTAAAAT-3', CFTR reverse: 5'-AGTTGGCACGCTTTGATGACACTCC-3'; ROCK1 forward: 5'-GGGAGGTGAGATTAGGGCGA-3', ROCK1 reverse: 5'-CAACTGGTGCCACAGTGTCT-3'; CALM3 forward: 5'-CCGTGTTTTTGACAAGGATGG-3', CALM3 reverse: 5'-TCATCCACTTCCTCGTCGGT-3'; $M Y L K$ forward: 5'-GGGACTCAGTGGACAAGACG-3', MYLK reverse: 5'-CCTTGGGCTCCTCAGGTTTC-3'; ACTA2 forward: 5'-CGGGAGAAGATGACCCAGATTAT-3', ACTA2 reverse: 5'-CAGTCGTACGTCCAGAGGC-3'; MYL6 forward: 5'-TATGAAGCGTTTGTGAGGCATATC-3', MYL6 reverse: 5'-TTCAGGGCTACACACACCGT-3'; and RPL13 forward: 5'-TACCGCTCCAAGCTCATCCT-3', RPL13 reverse: 5'-CACGTTCCGTATGGGCATGA-3'. The cDNA was amplified in the 7900HT Fast Real-Time PCR System (Applied Biosystems). Relative mRNA expression was calculated using the $\Delta \Delta \mathrm{Ct}$ method (51). Individual data were normalized against the housekeeping gene ribosomal protein L13. Results are presented as fold change in expression over the average of the WT controls.

Library preparation and sequencing. Library preparation and sequencing were conducted in the Iowa Institute of Human Genomics core facility at the University of Iowa. Briefly, an Illumina TruSeq Stranded Total RNA kit (Illumina RS-122-2201) was used to isolate rRNA-depleted RNA. The samples were then reverse transcribed to create $\mathrm{cDNA}$. The cDNA was fragmented, blunt-ended, and ligated to indexed adaptors. Following quantification of the cDNA generated for the library, the samples were clustered and loaded equally over two lanes on an Illumina HiSeq 2000 Sequencing system (Illumina Inc.), which generated on average $>20$ million paired reads of $100 \mathrm{bp}$.

Data records. FASTQ files containing the raw RNA-seq reads were deposited in the National Center for Biotechnology Information (NCBI) Sequence Reads Archive (SRP108562), with an accompanying BioProject ID (PRJNA388808).

Filtering and mapping of sequence reads. Quality control, trimming, and mapping were performed with the Galaxy platform (52). Initially, the FastQC tool was applied to the raw sequence data followed by FastQ Trimmer (52). As a result, $15 \mathrm{bp}$ were trimmed from the $5^{\prime}$ end and $10 \mathrm{bp}$ from the $3^{\prime}$ end of each raw sequence read. Sequences containing $75 \mathrm{bp}$ and a Phred quality score of more than 20 were used in the gene expression analysis. Using the Bowtie short read aligner with no more than two mismatches (53), the filtered sequences were mapped onto the pig genome Sus scrofa 10.2., obtained from the NCBI database. Approximately $82 \%$ of the reads were mappable to genome and $76 \%-77 \%$ reads were paired. The ensembl annotation (iGenomes, Illumina) of the $S$. scrofa genome, along with the number of reads mapped within each annotated transcript, were used to calculate the level of transcription for each gene. The Cufflinks tool was used to estimate the relative abundances of the transcript reads for each gene (54). For comparison of gene expression between WT and CF samples, the read counts were normalized based on the number of fragments per kilobase of coding sequence per million mapped reads (FPKM).

Differential gene expression. The differential gene expression between WT and CF was assessed using the CuffDiff tool (54). In brief, the total read count for each gene was calculated by combining the expression data from all replicates into either WT or CF groups and testing for differentially expressed transcripts present in all 4 replicates within the group. FPKM values were expressed in $\log _{2}$ (FPKM) to allow for statistical comparisons. As a result, $\log _{2}$ fold change in abundance of each transcript was obtained by $\log _{2}$ (FPKM [CF]/FPKM [WT]). Cuffdiff calculated the abundance of the transcripts listed and tested concurrently for differential expression. $Q$ values of less than 0.05 and fold change \pm 2 in the Cuffdiff output were selected as the criteria for significant difference. Significant differential expression was determined in genes with $Q<0.05$ and fold change \pm 2 .

Functional GO and pathway analysis. Biological functions and interactions of the genes and proteins differentially expressed were determined using MetaCore (Thomson Reuters), IPA (Qiagen), and the Database for Annotation Visualization and Integrated Discovery (DAVID) (55). The MetaCore and IPA pathways were 
used for pathway and network analysis, and the DAVID knowledge base was used for functional clustering. The Cytoscape software (http://www.cytoscape.org/), including a ClueGo plug-in, was used to visualize GO terms and pathways in functionally organized networks. This allows for examination of the relations between the biological terms based on the similarity of their linked gene/proteins. Using Cytoscape, the differentially expressed gene list obtained from CF airway smooth muscle was uploaded in ClueGO. For the enrichment of biological terms and groups, we used the following settings: the $\kappa$ statistics score threshold was set to 0.3 . Other analysis parameters included GO level intervals of $3-15$, and the number of associated proteins was 9. All functional GO and pathways analyses were performed using annotated genes that also had human orthologs recognized in the respective databases.

Airway smooth muscle tissue and cells. Tracheas from newborn non-CF $\left(\mathrm{CFTR}^{+/+}\right.$and $\left.\mathrm{CFTR}^{+/-}\right)$or $\mathrm{CFTR}^{-/-}$ pigs were excised and placed in modified Krebs Solution containing $120.8 \mathrm{mM} \mathrm{NaCl}, 5.9 \mathrm{mM} \mathrm{KCl}, 0.2 \mathrm{mM}$ $\mathrm{CaCl}_{2}, 1.2 \mathrm{mM} \mathrm{MgCl}_{2}, 1.2 \mathrm{mM} \mathrm{NaH}_{2} \mathrm{PO}_{4}, 2 \mathrm{mM} \mathrm{NaHCO}_{3}, 11 \mathrm{mM}$ d-glucose, and $10 \mathrm{mM} \mathrm{HEPES}, \mathrm{pH} 7.4$. The trachealis muscle was dissected by removing the cartilage and epithelial layers. For force measurements, trachealis smooth muscle strips were hung in a tissue perfusion system (Radnoti LLC). For cell isolation, the remaining medial layers were minced and digested using a papain dissociation system (Worthington) based upon previous studies (56). The minced smooth muscle was suspended in Earle's balanced salt solution containing papain $(10 \mathrm{U} / \mathrm{ml})$ and DNase $(1,000 \mathrm{U} / \mathrm{ml})$ and incubated at $37^{\circ} \mathrm{C}$ for 1 hour. Collagenase $(0.5 \mathrm{mg} / \mathrm{ml})$ was added, and after 1 hour the mixture triturated. Primary cells were grown to confluence in a 1:1 mixture of DMEM and Ham's F12 on fibrinogen-coated 6-well plates. All solutions contained 100 U/ $\mathrm{ml}$ penicillin and $0.1 \mathrm{mg} / \mathrm{ml}$ streptomycin. Primary culture cells were allowed to grow for 8-10 days until confluent. Positive immunostaining with the polyclonal anti- $\alpha$-smooth muscle actin antibody (clone $1 \mathrm{~A} 4$, abcam) confirmed the presence of airway smooth muscle cells and lack of staining with the monoclonal anti-fibroblast surface protein antibody (clone 1B10, abcam) excluded the presence of fibroblasts.

Protein phosphorylation profiling of airway smooth muscle. The Phospho Explorer antibody microarray (Full Moon BioSystems) contains 1,318 antibodies in duplicate. The assay was performed using cultured passage 1-2 WT and CF airway smooth muscle cells. Proteins from both genotypes were collected, biotinylated, and incubated with the antibody array as per the manufacturer's instructions. Slides were analyzed using an Axon 4000B microarray scanner (Molecular Devices). Individual antibody matrix dots underwent global mean normalization to housekeeping signal intensity with subsequent normalization of CF to WT signal intensities to derive relative expression values. Positive values correspond to highly expressed proteins or phosphorylation sites with increased phosphorylation, respectively, in $C F T R^{-/-}$airway smooth muscle. Negative values refer to under expressed proteins or phosphorylation sites with decreased phosphorylation. For quantified ELISA studies, select proteins (PYK2, the p38 regulatory unit of PI3K, and the p85 regulatory unit of MAPK) were assayed in donor pools of 4, on 3 separate occasions, for a total of 12 donors.

LINCS analysis. To identify chemical modulators that negatively correlated with CFTR knockout in airway smooth muscle, we queried LINCS, supported by the NIH Common Fund (57). LINCS represents a database of gene expression data collected from numerous human cells treated with chemical compounds. This novel mRNA expression profiling technique was used to compare the $C F T R^{-/-}$airway smooth muscle transcriptional signature across multiple cell and perturbation types in the database. In total, the signature was queried against 15 human cell lines responding to 16,425 chemical reagents in the LINCS data set. A connectivity score was assigned to each of the expression profiles from the perturbations based on a weighted Kolmogorov-Smirnov statistic.

LINCS compound associations with disease states. To define associations of LINCS compounds with diseases, a search for the terms, including the compound name as identified by LINCS, and a disease (asthma, chronic kidney disease, and diabetes) was performed. PubMed was used to search for associations with the inclusion criteria limited to randomized controlled trials, cohort studies, case-control studies, and any reviews with an implicit or an explicit mention of the queried compound and either asthma, chronic kidney disease, and/or diabetes. If an article was found using these criteria the compound was indicated as an association. The top 60 compounds were used for analysis and compared to 60 control compounds that LINCS predicted to have no association with the CF signature (null group, enrichment score $=0$ ). For differences between the top 60 compounds and the null group of compounds, the odds of the top 60 compounds being associated with a disease state were compared with those of the null compound group by odds ratio; $95 \%$ confidence intervals (CI) were computed along with $P$ values for the null hypothesis that the odds ratio is 1 . 
Measurement of airway smooth muscle force generation. Freshly harvested tracheal rings were bubbled in Ringer's solution overnight in the presence of either $10 \mu \mathrm{M}$ NVP-TAE684 (Selleck Chemical) or DMSO vehicle. The next day, airway smooth muscle strips $(\sim 1.5 \mathrm{~mm} \times \sim 3 \mathrm{~mm})$ from the trachealis muscle were mounted along the trachealis muscle orientation in individual muscle baths (Radnoti Glass) and force generation was quantified as previously described (11). The smooth muscle force from NVP-TAE684- or DMSO-pretreated strips was determined by obtaining concentration-response curves to acetylcholine $\left(10^{-9}\right.$ to $10^{-2} \mathrm{M}$, Sigma-Aldrich) in the muscle bath.

MLC protein extraction and Western blot analysis. WT airway smooth muscle strips pretreated with either NVP-TAE684 or DMSO were hung at reference length in an organ perfusion bath and allowed to equilibrate for 1 hour in Krebs buffer. Following equilibration, strips were treated with an $\mathrm{EC}_{50}$ dose of acetylcholine $(\sim 10 \mu \mathrm{M})$. Acetone prechilled on dry ice was used to freeze the tissue while hanging. Phosphorylated and nonphosphorylated MLC levels were then determined as previously described (11).

Precision-cut lung slice preparation. Precision-cut lung slices were isolated as previously described from WT newborn pigs (11). For luminal diameter changes in response to methacholine, lung slices were pretreated overnight with either NVP-TAE684 $(10 \mu \mathrm{M}$, Selleck Chemical) or DMSO $(0.1 \%$ final concentration) and then mounted in the perfusion chamber and perfused with HBSS for 5 minutes. Lung slices were perfused with increasing concentrations of methacholine (0-10 $\mu \mathrm{M}$, Sigma-Aldrich) to induce airway contraction in the lung slice. For all precision-cut lung slice experiments, maximal airway contraction was analyzed for each dose by lumen area measurements performed with ImageJ (NIH). Paired comparisons were performed using sequentially cut lung slices pretreated with either NVP-TAE684 or DMSO.

Measurement of in vivo airway resistance. WT mice ( $\sim 3$ month, $\sim 20 \mathrm{~g})$ were pretreated with either the PYK2 inhibitor NVP-TAE684 (100 $\mu 1$ of $5 \mathrm{mM}, 1 \%$ DMSO) or vehicle (1\% DMSO) via intraperitoneal injections daily for 3 days prior to and including the day of pulmonary measurements. Mice were anesthetized with ketamine and xylazine. Following tracheotomy, an 18-gauge cannula was inserted and securely tied with 4.0 braided silk. The animals were then paralyzed with rocuronium bromide and mechanically ventilated with a computer-controlled small-animal ventilator (FlexiVent, SCIREQ) using a tidal volume of $8 \mathrm{ml} / \mathrm{kg}$ at a rate of 180 breaths per minute and PEEP of $3.5 \mathrm{~cm} \mathrm{H}_{2} 0$. Pulmonary resistance was measured by the computer-controlled ventilator by interrupting ventilation and imposing broadband low-frequency oscillatory waveforms and then resuming ventilation. After measuring baseline resistance, mice underwent airway challenge with normal saline and increasing dosages of methacholine by aerosol challenge of 20 $\mu 1$ of methacholine solutions ranging from 0.3 to $100 \mathrm{mg} / \mathrm{ml}$. Measurements of resistance were obtained for each dose at 10-second intervals for 2 minutes, and then the next methacholine dose was administered.

PYK2 Western blot analysis. Cells were lysed with modified RIPA lysis buffer (50 mM Tris, pH 7.5, 150 $\mathrm{mM} \mathrm{NaCl}, 0.3 \%$ sodium deoxycholate, $0.1 \% \mathrm{NP}-40,10 \%$ glycerol, $1.5 \mathrm{mM} \mathrm{MgCl}, 1 \mathrm{mM}$ EDTA, $0.2 \mathrm{mM}$ EGTA, $20 \mathrm{mM} \mathrm{NaF}, 25 \mu \mathrm{M} \mathrm{ZnCl}_{2}, 1 \mathrm{mM} \mathrm{NaVO}_{4}, 1 \mathrm{mM}$ PMSF, complete protease inhibitor tablet [Roche] and Phosphatase Inhibitor Cocktail [Thermo Fischer Scientific]). Immunoprecipitation was carried out at $4^{\circ} \mathrm{C}$ by incubating cell lysates overnight with rabbit anti-PYK2 antibody (3292, Cell Signaling Technology), followed by an incubation for 1 hour with protein A-Dynabeads (Life Technologies). Immunoprecipitates were washed 3 times in lysis buffer without protease inhibitors. The beads were resuspended in SDS-PAGE sample buffer, boiled for 5 minutes, and resolved by SDS-PAGE. Western blotting was performed with rabbit anti-PYK2 and rabbit anti-Phospho-Pyk2 (Tyr402) antibody (Cell Signaling Technology, 3291), using IRdye secondary antibodies on an Odyssey imaging system (LI-COR Biosciences).

Protein phosphorylation profiling of airway smooth muscle. A custom antibody array (Full Moon Biosystems) was used for select proteins (PYK2, the p38 regulatory unit of PI3K, and the p85 regulatory unit of MAPK). The assay was performed using cultured passage of 1-2 WT and CF airway smooth muscle cells. Proteins from both genotypes were collected, biotinylated, and incubated with the antibody array as per the manufacturer's instructions. A total of 4 donors were pooled onto 1 slide, and a minimum of 3 slides were run for statistical analysis ( $n=3$ slides, representing 12 animal donors). Slides were analyzed using an Axon 4000B microarray scanner (GenePix). Individual antibody matrix dots underwent global mean normalization to housekeeping signal intensity with subsequent normalization of $\mathrm{CFTR}^{-/-}$to WT signal intensities to derive relative expression values. Positive values correspond to highly expressed proteins or phosphorylation sites with increased phosphorylation, respectively, in $\mathrm{CFTR}^{-/-}$airway smooth muscle. Negative values refer to underexpressed proteins or phosphorylation sites with decreased phosphorylation. 
In vitro PYK2 kinase assay. The specific PYK2 kinase activity in the presence and absence of NVPTAE684 was measured using a Universal Kinase Activity Kit (R\&D Systems). The assay measures the inorganic phosphate that is released from ADP via substrate phosphorylation by PYK2. Different concentrations of recombinant human PYK2 $(0.5$ and $2 \mu \mathrm{g} / \mathrm{ml}, \mathrm{R} \& \mathrm{D}$ Systems $)$ were incubated in the presence of $200 \mu \mathrm{M}$ ATP and $1 \mu \mathrm{M}$ concentrations of a protein tyrosine kinase substrate (poly Glu,Tyr 4:1, Sigma-Aldrich) for 10 minutes at room temperature. Inorganic phosphate was used for standard curve determination, and ADP was used as a positive control. The reaction was terminated by application of malachite green. The optical density was measured at $620 \mathrm{~nm}$.

Statistics. Unless otherwise noted, results are expressed as mean \pm SEM or mean only. For analyses that compared two groups, we used a paired or unpaired 2-tailed Student's $t$ test. For odds ratio calculations, categorical variables were analyzed by $\chi^{2}$. For time-course and drug-dose curves, the analysis used a 4-parameter logistic regression algorithm (sigmoidal curve fit) to fit. For multiple comparisons, we used a 1-way ANOVA, followed by Dunnett's test. We considered $P<0.05$ statistically significant.

Study approval. All animal studies were reviewed and approved by the University of Iowa Animal Care and Use Committee.

\section{Author contributions}

DPC and DAS conceived and designed research, prepared figures, and drafted the manuscript; DPC, RJA, KZ, MRS, NDG, and DAS performed experiments; DPC, RJA, KZ, BD, DKM, KFA, and DAS analyzed data; DPC, KZ, DKM, and DAS interpreted results of experiments; and DPC, RJA, KZ, BD, MRS, NDG, DKM, KFA, and DAS edited and revised the manuscript and approved the final version.

\section{Acknowledgments}

We thank M. Abou Alaiwa, P. McCray, L. Ostedgaard, L. Powers, L. Reznikov, S. Roach, T. Ruggle, P. Taft, K. Webber, M. Welsh, J. Zabner, and the University of Iowa Office of Animal Resources for excellent assistance, advice, and discussions. This work was supported by an Early Excellence Award from the American Asthma Foundation (to DAS), the NIH (HL091842, HL051670, HL117744, T32 GM007337, T32 HL007638), and the Cystic Fibrosis Foundation (Research Development Program). This work utilized the Leica LMD7000 Laser Capture Microdissection System in the University of Iowa Central Microscopy Facilities, which was purchased with funding from NIH Shared Instrument Grant S10 OD016316.

Address correspondence to: David A. Stoltz, Lung Biology and Cystic Fibrosis Research Center, Pappajohn Biomedical Institute, University of Iowa, 6322 PBDB, 169 Newton Road, Iowa City, Iowa 52242, USA. Phone: 319.384.1107; Email: david-stoltz@uiowa.edu.

1. Nielsen AO, Qayum S, Bouchelouche PN, Laursen LC, Dahl R, Dahl M. Risk of asthma in heterozygous carriers for cystic fibrosis: A meta-analysis. J Cyst Fibros. 2016;15(5):563-567.

2. Adam RJ, et al. Acute administration of ivacaftor to people with cystic fibrosis and a G551D-CFTR mutation reveals smooth muscle abnormalities. JCI Insight. 2016;1(4):e86183.

3. Robert R, et al. Regulation of the cystic fibrosis transmembrane conductance regulator channel by beta-adrenergic agonists and vasoactive intestinal peptide in rat smooth muscle cells and its role in vasorelaxation. J Biol Chem. 2004;279(20):21160-21168.

4. Robert R, Norez C, Becq F. Disruption of CFTR chloride channel alters mechanical properties and cAMP-dependent Cl- transport of mouse aortic smooth muscle cells. J Physiol (Lond). 2005;568(Pt 2):483-495.

5. Vandebrouck C, et al. Evidence that CFTR is expressed in rat tracheal smooth muscle cells and contributes to bronchodilation. Respir Res. 2006;7:113.

6. Robert R, Savineau JP, Norez C, Becq F, Guibert C. Expression and function of cystic fibrosis transmembrane conductance regulator in rat intrapulmonary arteries. Eur Respir J. 2007;30(5):857-864

7. Michoud MC, et al. Role of the cystic fibrosis transmembrane conductance channel in human airway smooth muscle. Am J Respir Cell Mol Biol. 2009;40(2):217-222.

8. McCuaig S, Martin JG. How the airway smooth muscle in cystic fibrosis reacts in proinflammatory conditions: implications for airway hyper-responsiveness and asthma in cystic fibrosis. Lancet Respir Med. 2013;1(2):137-147.

9. Wallace HL, Southern KW, Connell MG, Wray S, Burdyga T. Abnormal tracheal smooth muscle function in the CF mouse. Physiol Rep. 2013;1(6):e00138.

10. Bazett M, Haston CK. Airway hyperresponsiveness in FVB/N delta F508 cystic fibrosis transmembrane conductance regulator mice. J Cyst Fibros. 2014;13(4):378-383.

11. Cook DP, et al. Cystic fibrosis transmembrane conductance regulator in sarcoplasmic reticulum of airway smooth muscle. Implications for airway contractility. Am J Respir Crit Care Med. 2016;193(4):417-426.

12. Stoltz DA, et al. Cystic fibrosis pigs develop lung disease and exhibit defective bacterial eradication at birth. Sci Transl Med. 
2010;2(29):29ra31.

13. Berridge MJ, Lipp P, Bootman MD. The versatility and universality of calcium signalling. Nat Rev Mol Cell Biol. 2000;1(1):11-21.

14. Yick CY, et al. Gene expression profiling of laser microdissected airway smooth muscle tissue in asthma and atopy. Allergy. 2014;69(9):1233-1240.

15. Pascoe CD, Swyngedouw NE, Seow CY, Paré PD. Gene expression in asthmatic airway smooth muscle: a mixed bag. Can J Physiol Pharmacol. 2015;93(2):137-143.

16. Woodruff PG. Gene expression in asthmatic airway smooth muscle. Proc Am Thorac Soc. 2008;5(1):113-118.

17. Gao N, Huang J, He W, Zhu M, Kamm KE, Stull JT. Signaling through myosin light chain kinase in smooth muscles. J Biol Chem. 2013;288(11):7596-7605.

18. Koziol-White CJ, et al. Inhibition of PI3K promotes dilation of human small airways in a rho kinase-dependent manner. $B r J$ Pharmacol. 2016;173(18):2726-2738.

19. Berend N, Salome CM, King GG. Mechanisms of airway hyperresponsiveness in asthma. Respirology. 2008;13(5):624-631.

20. Cohen JC, Larson JE, Killeen E, Love D, Takemaru K. CFTR and Wnt/beta-catenin signaling in lung development. BMC Dev Biol. 2008;8:70.

21. Than BL, et al. CFTR is a tumor suppressor gene in murine and human intestinal cancer. Oncogene. 2016;35(32):4179-4187.

22. Xu WM, et al. Defective CFTR-dependent CREB activation results in impaired spermatogenesis and azoospermia. PLoS ONE. 2011;6(5):e19120.

23. Bindea G, et al. ClueGO: a Cytoscape plug-in to decipher functionally grouped gene ontology and pathway annotation networks. Bioinformatics. 2009;25(8):1091-1093.

24. Li L, et al. REG $\gamma$ deficiency promotes premature aging via the casein kinase 1 pathway. Proc Natl Acad Sci USA. 2013;110(27):11005-11010.

25. Liu C, Su J, Yang F, Wei K, Ma J, Zhou X. Compound signature detection on LINCS L1000 big data. Mol Biosyst. 2015;11(3):714-722.

26. Takeda M, et al. Allergic airway hyperresponsiveness, inflammation, and remodeling do not develop in phosphoinositide 3-kinase gamma-deficient mice. J Allergy Clin Immunol. 2009;123(4):805-812.

27. Krymskaya VP. Targeting the phosphatidylinositol 3-kinase pathway in airway smooth muscle: rationale and promise. BioDrugs. 2007;21(2):85-95.

28. Lee KS, Lee HK, Hayflick JS, Lee YC, Puri KD. Inhibition of phosphoinositide 3-kinase delta attenuates allergic airway inflammation and hyperresponsiveness in murine asthma model. FASEB J. 2006;20(3):455-465.

29. Davis MI, et al. Comprehensive analysis of kinase inhibitor selectivity. Nat Biotechnol. 2011;29(11):1046-1051.

30. Galkin AV, et al. Identification of NVP-TAE684, a potent, selective, and efficacious inhibitor of NPM-ALK. Proc Natl Acad Sci USA. 2007;104(1):270-275

31. Sanderson MJ. Exploring lung physiology in health and disease with lung slices. Pulm Pharmacol Ther. 2011;24(5):452-465.

32. Park SY, Avraham HK, Avraham S. RAFTK/Pyk2 activation is mediated by trans-acting autophosphorylation in a Src-independent manner. J Biol Chem. 2004;279(32):33315-33322.

33. Lev S, et al. Protein tyrosine kinase PYK2 involved in $\mathrm{Ca}(2+)$-induced regulation of ion channel and MAP kinase functions. Nature. 1995;376(6543):737-745.

34. Duan Y, Learoyd J, Meliton AY, Clay BS, Leff AR, Zhu X. Inhibition of Pyk2 blocks airway inflammation and hyperresponsiveness in a mouse model of asthma. Am J Respir Cell Mol Biol. 2010;42(4):491-497.

35. Schaller MD. Calcium-dependent Pyk2 activation: a role for calmodulin? Biochem J. 2008;410(3):e3-e4.

36. Mills RD, Mita M, Nakagawa J, Shoji M, Sutherland C, Walsh MP. A role for the tyrosine kinase Pyk2 in depolarization-induced contraction of vascular smooth muscle. J Biol Chem. 2015;290(14):8677-8692.

37. Bhattachariya A, et al. PYK2 selectively mediates signals for growth versus differentiation in response to stretch of spontaneously active vascular smooth muscle. Physiol Rep. 2014;2(7):e12080

38. Hirota S, Trimble N, Pertens E, Janssen LJ. Intracellular Cl- fluxes play a novel role in Ca2+ handling in airway smooth muscle. Am J Physiol Lung Cell Mol Physiol. 2006;290(6):L1146-L1153.

39. Gallos G, Yim P, Emala CW. Chloride in airway smooth muscle: the ignored anion no longer? Am J Physiol Lung Cell Mol Physiol. 2012;302(8):L733-L735.

40. Kitamura K, Yamazaki J. Chloride channels and their functional roles in smooth muscle tone in the vasculature. Jpn J Pharmacol. 2001;85(4):351-357.

41. Nelson MT, Conway MA, Knot HJ, Brayden JE. Chloride channel blockers inhibit myogenic tone in rat cerebral arteries. J Physiol (Lond). 1997;502 (Pt 2):259-264.

42. Pollock NS, Kargacin ME, Kargacin GJ. Chloride channel blockers inhibit Ca2+ uptake by the smooth muscle sarcoplasmic reticulum. Biophys J. 1998;75(4):1759-1766.

43. Grossi M, et al. Pyk2 inhibition promotes contractile differentiation in arterial smooth muscle. J Cell Physiol. 2017;232(11):3088-3102.

44. Rogers CS, et al. The porcine lung as a potential model for cystic fibrosis. Am J Physiol Lung Cell Mol Physiol. 2008;295(2):L240-L263.

45. Stoltz DA, Meyerholz DK, Welsh MJ. Origins of cystic fibrosis lung disease. N Engl J Med. 2015;372(4):351-362.

46. Stoltz DA, et al. Intestinal CFTR expression alleviates meconium ileus in cystic fibrosis pigs. J Clin Invest. 2013;123(6):2685-2693.

47. Ostedgaard LS, et al. The $\Delta$ F508 mutation causes CFTR misprocessing and cystic fibrosis-like disease in pigs. Sci Transl Med. 2011;3(74):74ra24.

48. Norez C, Jayle C, Becq F, Vandebrouck C. Bronchorelaxation of the human bronchi by CFTR activators. Pulm Pharmacol Ther. 2014;27(1):38-43.

49. Rogers CS, et al. Disruption of the CFTR gene produces a model of cystic fibrosis in newborn pigs. Science. 2008;321(5897):1837-1841.

50. Rogers CS, et al. Production of CFTR-null and CFTR-DeltaF508 heterozygous pigs by adeno-associated virus-mediated gene targeting and somatic cell nuclear transfer. J Clin Invest. 2008;118(4):1571-1577. 
51. Silver N, Best S, Jiang J, Thein SL. Selection of housekeeping genes for gene expression studies in human reticulocytes using real-time PCR. BMC Mol Biol. 2006;7:33.

52. Blankenberg D, et al. Manipulation of FASTQ data with Galaxy. Bioinformatics. 2010;26(14):1783-1785.

53. Langmead B. Aligning short sequencing reads with Bowtie. Curr Protoc Bioinformatics. 2010; Chapter 11:Unit 11.7.

54. Trapnell C, et al. Transcript assembly and quantification by RNA-Seq reveals unannotated transcripts and isoform switching during cell differentiation. Nat Biotechnol. 2010;28(5):511-515.

55. Huang da W, Sherman BT, Lempicki RA. Systematic and integrative analysis of large gene lists using DAVID bioinformatics resources. Nat Protoc. 2009;4(1):44-57.

56. Townsend EA, Sathish V, Thompson MA, Pabelick CM, Prakash YS. Estrogen effects on human airway smooth muscle involve cAMP and protein kinase A. Am J Physiol Lung Cell Mol Physiol. 2012;303(10):L923-L928.

57. Lamb J, et al. The Connectivity Map: using gene-expression signatures to connect small molecules, genes, and disease. Science. 2006;313(5795):1929-1935. 